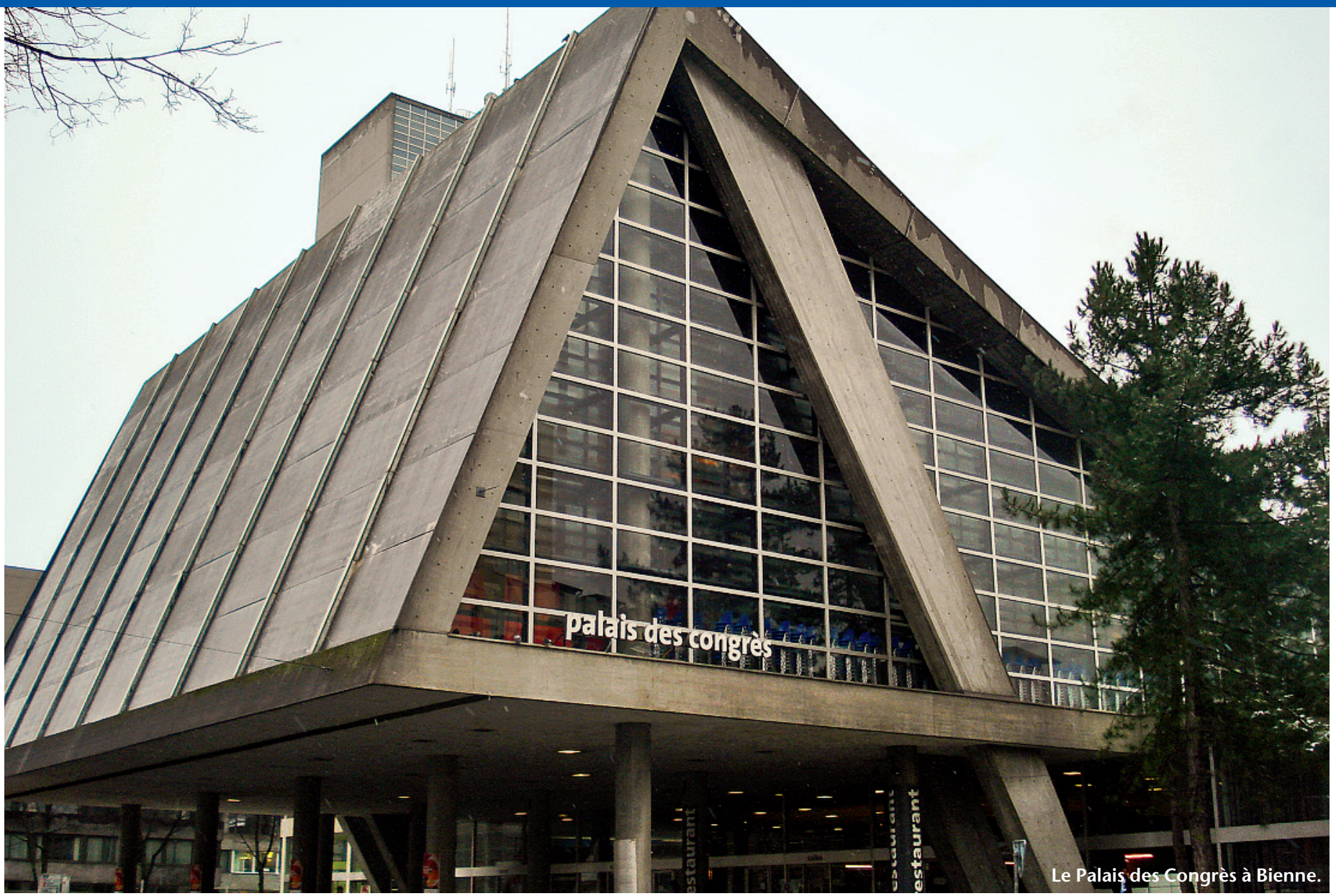

\title{
Procès-verbal de la Chambre médicale extraordinaire
}

Erika Flückiger,

responsable des Services

Un glossaire des abréviations les plus usitées figure à la page 163.

\section{Salutations, communications, constitu- tion du Bureau}

Jacques de Haller, président de la FMH, ouvre la séance à 9 h36 en constatant que le quorum, fixé à la moitié des délégués ayant droit de vote, est atteint. Il souhaite la bienvenue aux délégués présents et aux hôtes de ce jour: Jon Caflish pour le point 8.1.1 Pédiatrie du développement, Franz Caduff et Ulrich Schnyder pour le point 8.1.2 Psychiatrie de consultation-liaison, Roland Schwarz et Philippe Rheiner, membres de la commission de gestion (CdG), Bruno Kesseli, rédacteur en chef du Bulletin des médecins suisses (BMS), Reinhard Kunz, directeur de FMH Services et Kurt Schreier, président désigné de la Société de médecine du canton de Schwyz. Le président prend simultanément congé de Thomas Keller, présent aujourd'hui pour la dernière fois en qualité de président de la société cantonale schwytzoise, et le remercie de sa longue activité. Après avoir donné quelques informations techniques et organisationnelles, il désigne les scrutateurs suivants: Pierre Vallon/ SSPP, Markus Reber/LU, Philippe Rheiner/GE et CdG, Mitsouko Kondo Oestreicher/SPTC, Günther Gruber/ SSRO, Carlo Moll/AMDHS, Urs Stoffel/ZH, Michaël Hofer/SSP, Fabrice Dami/ASMAC, Christoph Bosshard/ASMAC. Ces personnes forment le Bureau de la séance de ce jour, conjointement avec le président, les deux vice-présidents et le secrétaire général. Le président fixe à 17 h00 l'heure après laquelle il ne sera plus possible de prendre des décisions ou de voter.

Après l'envoi de la convocation, les propositions suivantes sont parvenues à la FMH en vue de leur inscription à l'ordre du jour: proposition Maurice Stauffacher/VD, point 4.2, proposition Markus Reber/LU, point 4.3, proposition Christoph Ramstein/VEDAG, point 6.4. Toute modification de l'ordre du jour nécessite un vote requérant une majorité des deux tiers. Deux autres 
propositions ont été déposées en début de séance: celle de Christoph Ramstein/VEDAG sera traitée sous le point 2, et celle de Peter Wiedersheim/SG sous le point 12/Divers. La Chambre médicale approuve l'ordre du jour ainsi modifié sans opposition ni abstention.

\section{Examen et approbation des objectifs politiques et stratégiques proposés par le Comité central et l'Assemblée des délégués}

Concernant les objectifs politiques et stratégiques prévus pour la période administrative 2008-2012, il s'agit de définir dans les grandes lignes les activités de la FMH, resp. le mandat du Comité central. Le but et les tâches de la FMH sont fixés aux articles 2 et 3 des statuts. Il incombe au Comité central de sélectionner les objectifs et les tâches qui doivent figurer en priorité dans le programme de la législature en fonction de la situation politique. Lors de la Chambre médicale ordinaire de mai dernier, le Comité central a déjà soumis aux délégués quelques objectifs stratégiques. Le nouveau Comité central a repris ces travaux. Les objectifs qu'il a mis au point ont été traités à l'Assemblée des délégués de sorte que la Chambre médicale dispose aujourd'hui d'un document définitif. Conformément aux statuts, ces objectifs sont mis à jour chaque année en vue de leur adoption par la Chambre médicale ordinaire.
Pour la séance de ce jour, Christoph Ramstein/ $V E D A G$ a déposé la proposition de renvoi $\mathrm{n}^{\circ} 2$ relative au point 2 . Il estime que ces objectifs sont en quelque sorte la carte de visite de la FMH vis-à-vis de l'extérieur. Le document actuel contient la stratégie prévue pour une période administrative dont la première année est déjà presque terminée. Il regrette le manque de visions et souhaite plutôt un document allant dans la direction d'une stratégie associative proprement dite. Le style et la formulation choisie sont extrêmement importants pour que les parlementaires et les assureurs comprennent correctement nos objectifs. De ce fait, il souhaite que ce document soit renvoyé à l'Assemblée des délégués pour qu'elle en affine le style et le contenu en s'adjoignant un politicien expérimenté.

Le président demande à la Chambre médicale de voter sur l'inclusion de cette proposition dans l'ordre du jour. Les délégués l'approuvent par 98 voix contre 26 , la majorité des deux tiers étant ainsi atteinte. Dans la discussion animée qui suit, quelques délégués sont agacés de ne pouvoir débattre aujourd'hui d'un "programme de gouvernement» pour les trois années restantes après que la stratégie a pris tant de temps à être élaborée. D'autres délégués expriment l'impossibilité qu'ils ressentent de défendre une stratégie vers l'extérieur qui ne soit pas adoptée par tous; ils aimeraient donc tout d'abord organiser une large

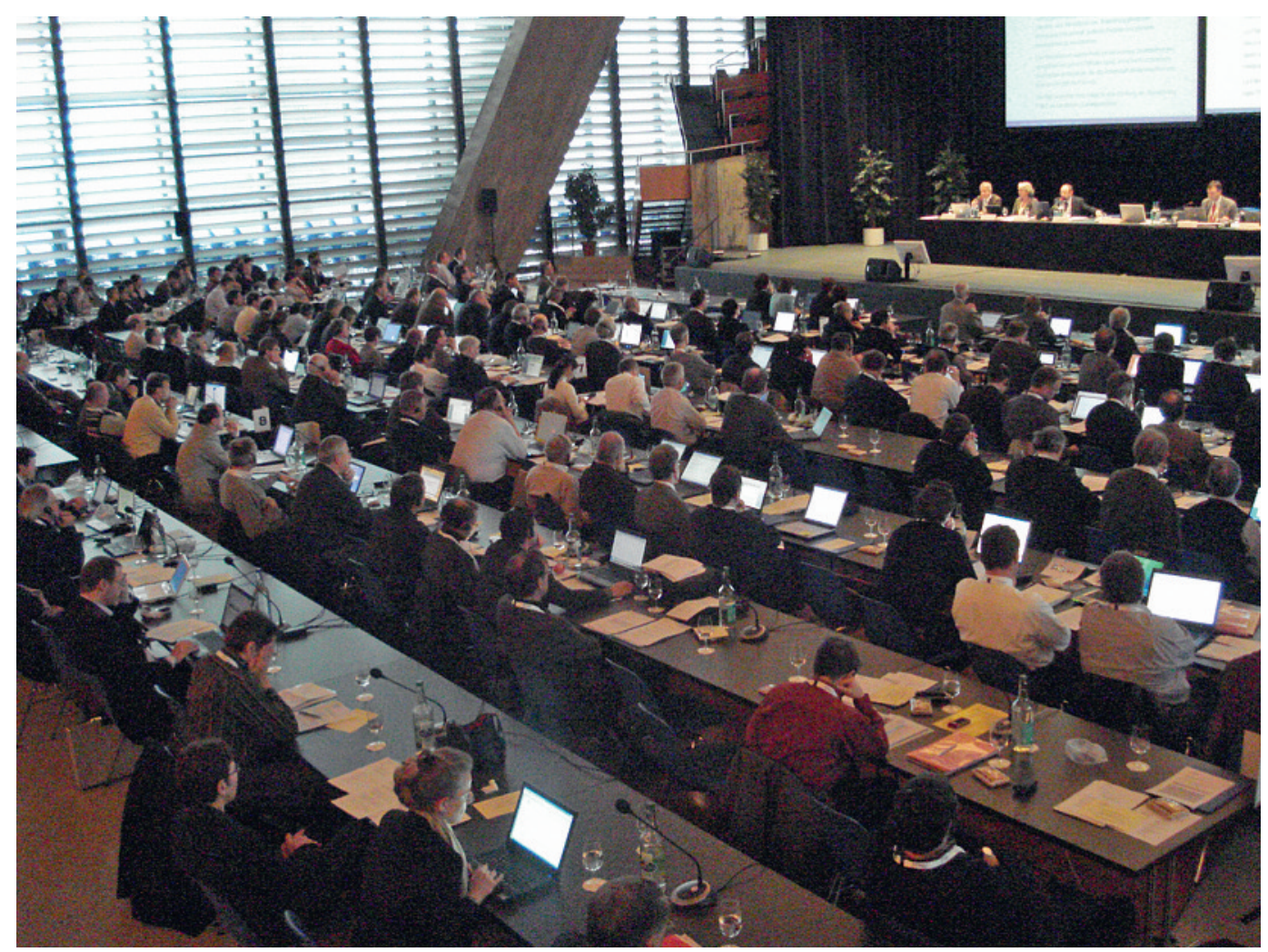

La Chambre médicale réunie. 
consultation auprès de la base. D'autres encore estiment que ce document est très positif. Finalement, il ressort de la discussion que les délégués sont partis de points de vue différents, ce qui a conduit à un malentendu. Les objectifs stratégiques et politiques visent à la fois à donner une structure à la présente période administrative et à servir de base à une stratégie en constante évolution. Le président demande aux délégués de se prononcer sur la proposition C. Ramstein $n^{\circ} 2$ relative au point 2 et il précise qu'accepter la proposition reviendra à ne pas entrer en matière sur le document «Objectifs stratégiques pour la législature 2008 à 2012», alors que la refuser signifiera débattre du document actuel.

C. Ramstein demande la parole pour annoncer qu'il retire sa proposition, compte tenu des explications qui viennent d'être données. La Chambre médicale peut donc maintenant examiner le contenu des objectifs fixés.

Le président commence la présentation des objectifs en précisant que le point 2.3 du document a glissé malencontreusement sous l'objectif 3 .

L'objectif 1 concerne la position que la FMH entend occuper en politique de santé. Hans Ulrich Iselin/AG souligne qu'il faut pour cela des médecins qui soient prêts à s'engager sur le plan politique.

L'objectif 2 concerne l'engagement de la FMH en faveur de la qualité des soins médicaux. Un délégué de la Société vaudoise de médecine (SVM) constate que nous disposons en Suisse d'un système de santé très performant et rentable. Pourquoi ne devrions-nous pas exiger un effort concret de la part de la Confédération, qui distribue aujourd'hui de l'argent aux banques, aux chemins de fer et à beaucoup d'autres, afin de maintenir le système de santé actuel et de l'améliorer? J. de Haller remercie pour cette remarque importante qu'il ne classe pas forcément sous le thème de la qualité mais éventuellement sous l'objectif 5. Pour Urs Stoffel/ZH, la promotion d'un label de qualité, qu'il considère être un objectif clair de la FMH, n'est pas totalement exempte de danger. Le président comprend cette remarque et souligne combien il sera important d'être hautement crédible dans ce domaine. Mais le fait est que le corps médical suisse fournit des prestations de qualité élevée et qu'il ne doit pas craindre de le communiquer.

Hans-Anton Vogel/AI-AR estime que ce point n'a pas de lien direct avec le thème de la qualité des soins médicaux, mais qu'il doit être traité sous forme d'objectif en tant que tel. Comme les avis sont partagés à ce sujet, le président demande aux délégués de voter pour savoir s'ils veulent laisser le point 2.3 à cet endroit ou le présenter de façon séparée dans le cadre d'un vote. Une claire majorité se prononce en faveur du maintien de ce point sous l'objectif 2 .

L'objectif 3 traite du rôle de la FMH dans la qualité de la formation professionnelle des médecins.

Pierre Meyrat/SO aimerait savoir si le point 3.1 ne se trouve pas en conflit avec le renforcement et l'autonomisation prévus de la Commission pour la formation postgraduée et continue (CFPC). Max Giger, président de la CFPC, et Hanspeter Kuhn, secrétaire général adjoint et responsable du service juridique de la $\mathrm{FMH}$, répondent par la négative à cette question.

L'objectif 4 concerne la relation médecinpatient. Il faut préserver la liberté thérapeutique, quel que soit le système (donc aussi dans les réseaux de médecins), et garantir le principe du libre choix du médecin tout en sachant que ce choix est limité dans les réseaux de médecins. Jürg Schlup/BE propose de débattre ici de la proposition de M. Stauffacher/VD dont l'inclusion dans l'ordre du jour sous le point 4/Révision partielle de la LAMal doit encore être votée. Dans sa proposition, Maurice Stauffacher/VD demande que «la Fédération des médecins suisses et son Comité central s'engagent pour une défense inconditionnelle de ce que l'on a convenu d'appeler «l'obligation de contracter», en particulier dans le cadre des réseaux de soins». A son avis, cette demande doit impérativement figurer dans la stratégie de la FMH parce qu'elle est l'une des pierres d'angle de notre système de santé et que le corps médical s'est clairement engagé dans ce

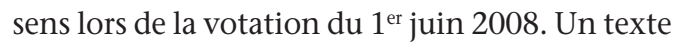
complémentaire peut être ajouté à l'objectif 4 , ou alors à l'objectif 7. J. de Haller le verrait plutôt sous l'objectif 7 et demande aux délégués vaudois de rédiger une phrase claire. La formulation relative au libre choix du médecin, telle qu'elle a été choisie sous l'objectif 4 , donne lieu à un débat contradictoire suite à l'intervention de Pierre Meyrat/SO qui exige le libre choix explicite du médecin pour le domaine de l'assurance obligatoire de base. Le président explique qu'on a choisi cette formulation tout à fait intentionnellement parce qu'elle est la seule qui permette de tenir compte de toutes les formes de systèmes de santé possibles, et notamment des réseaux de médecins. Une formulation trop rigide reviendrait à éliminer tout type de réseaux de médecins car, par définition, les patients renoncent dans une certaine mesure au libre de choix du médecin lorsqu'ils font partie d'un réseau. HP. Kuhn/secrétaire général adjoint attire l'attention sur le fait que les patients hospitalisés n'ont jamais le libre 


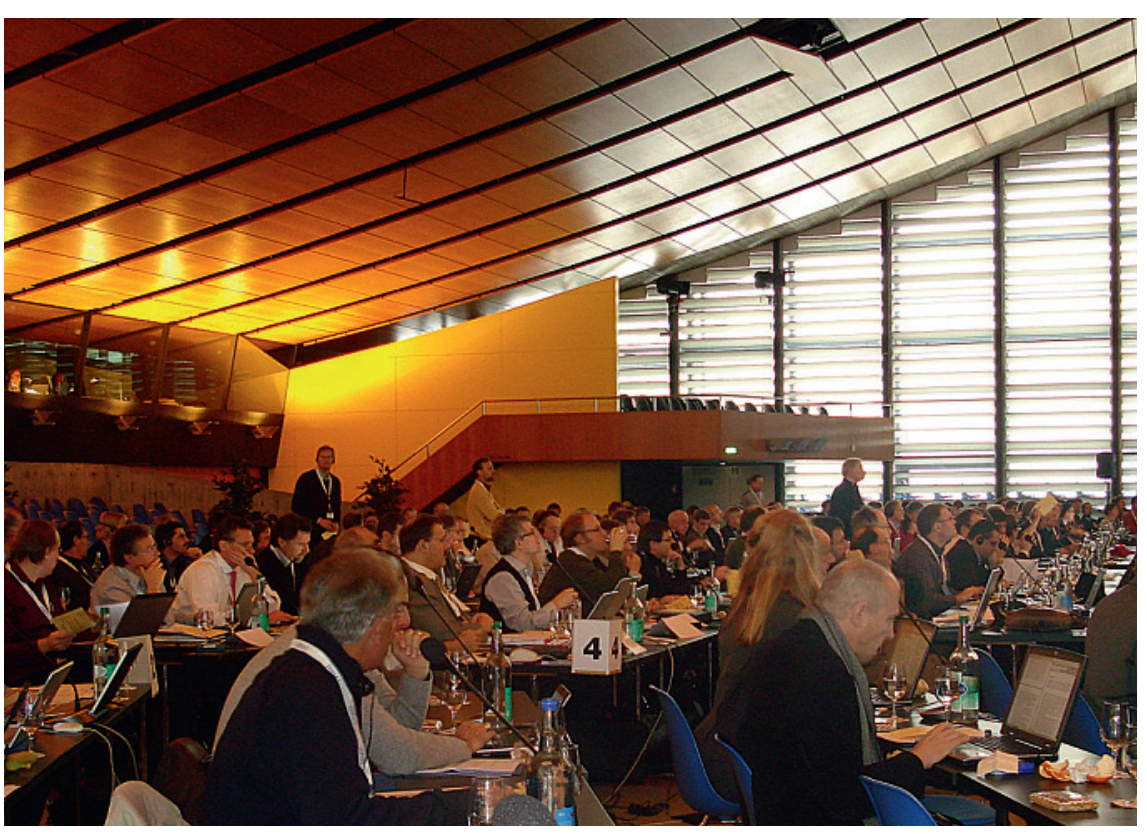

On procède au vote. ligne encore une fois que l'obligation de contracter est compatible avec les systèmes de Managed Care, contrairement au libre choix illimité du médecin.

L'objectif 5 concerne les relations de confiance entre le corps médical et le public.

Cet objectif ne donne lieu à aucune prise de parole.

L'objectif 6 concerne la défense des intérêts politico-professionnels des membres de la FMH. Beat Dubs/ZH regrette ici que la solidarité entre les membres de la FMH ne soit pas mentionnée. $J$. de Haller demande à $\mathrm{B}$. Dubs de mettre sa requête par écrit puis de la remettre au secrétaire général.

L'objectif 7 concerne l'engagement de la FMH pour une rémunération convenable des prestations médicales.

Le président demande à M. Stauffacher/SVM de présenter encore une fois sa demande: «La FMH s'engage pour une défense inconditionnelle de ce que l'on a convenu d'appeler «l'obligation de contracter», aussi dans le cadre des réseaux de soins». Le président ouvre le débat à ce sujet. Il estime personnellement que cette requête est difficile à défendre sur le plan politique car on ne pourra guère convaincre le Parlement d'obliger les assureurs de conclure des conventions avec tous les réseaux de médecins, mais il pense néanmoins que l'on peut l'introduire à cet endroit. La discussion qui suit est animée; certains délégués mettent en garde contre une trop forte réglementation dans le domaine des réseaux de médecins, ou voudraient même que l'expression «obligation de contracter» ne figure pas dans un document de base.

Le président soumet au vote la proposition de M. Stauffacher/SVM telle qu'il l'a lue en dernier. Cette proposition est rejetée par la majorité des délégués qui se prononcent ensuite sur la proposition de modification de H. Chaudhry/ASMAC relative à la proposition de M. Stauffacher/VD. La formulation «La Fédération des médecins suisses et son Comité central s'engagent pour une défense inconditionnelle de ce que l'on a convenu d'appeler l'<obligation de contracter»» est acceptée par une majorité de délégués et elle est ajoutée comme point supplémentaire à l'objectif 7 .

L'objectif 8 concerne le soutien accordé à la relève professionnelle. C. Ramstein/VEDAG trouve que cet objectif ne devrait pas figurer en dernière position dans le document stratégique, mais plutôt après l'objectif 3. C. Bosshard, président de l'ASMAC, remercie de ce soutien et confirme que la promotion de la relève devrait effectivement se trouver plus haut dans la liste des priorités. La Chambre médicale approuve cette proposition et peut fort bien intégrer ce complément ici. Il soude soins. M. Stauffacher/SVM propose d'ajoute sous le point 4 que «la défense de l'obligation de contracter représente une garantie pour le libre choix du médecin». Le président estime qu'on

choix du médecin. Pour rester crédibles, il ne faut pas poser des exigences qui ne correspondent pas Thomas Hofer/SSDV voit un autre conflit dans les points 3 et 4 de cet objectif. A son avis, l'exigence visant à ne pas restreindre l'accès aux soins médicaux pour les personnes économiquement médecin s'excluent mutuellement. Le président souligne qu'il s'agit justement ici d'une préoccuincitatifs économiques des systèmes de Managed Care ne vident de sa substance le principe du libre FMH réagit si fortement contre le modèle présenté par l'Helsana et santésuisse. Car si l'on donne aux (entiere liberté d' attirer les bons risque situation crainte par l'orateur précédent. Il s'agit donc de prévoir des incitations qui garantissent Homayon Chaudhry/ASMAC se réfère à sa proposition de modification $n^{\circ} 2$ (point 4.2) relative mande que la FMH s'engage sans réserve pour T'obligation de contracter, étant donné le manvotation du $1^{\text {er }}$ juin 2008, de maintenir sans conditions l'obligation de contracter aussi bien pour les cabinets individuels que pour les réseaux 
l'objectif 8 devient l'objectif 4 dans le document stratégique. C. Bosshard/ASMAC revient à l'objectif 7 et aimerait y mentionner que la FMH s'engage également en faveur d'une rémunération convenable des médecins hospitaliers salariés.

Michel Marchev/BE propose de mentionner explicitement sous l'objectif 7 - désormais objectif 8 - la promotion de la médecine de premier recours. Le président comprend cette demande mais il relève que l'on n'a pas voulu prioriser certaines disciplines à l'intérieur de la FMH car, outre les médecins de premier recours, d'autres disciplines ont aussi des problèmes.

Il soumet au vote la mention de la médecine de premier recours sous cet objectif, laquelle est rejetée par une claire majorité des délégués.

Andreas Haefeli/AG demande que l'objectif 8 soit complété par la phrase suivante: «La FMH s'engage pour des règlementations de politique économique et sanitaire aptes à garantir une fourniture de prestations efficace et économique.» Il motive sa proposition par le fait que les cabinets médicaux et les hôpitaux doivent toujours davantage faire face à des exigences de nature non médicale augmentant continuellement leurs coûts.

Le président demande l'avis de la Chambre médicale au sujet des propositions de C. Bosshard/ASMAC et d'A. Haefeli/AG. Celles-ci ne donnant lieu à aucune opposition, elles sont intégrées sous l'objectif 8 du document stratégique.

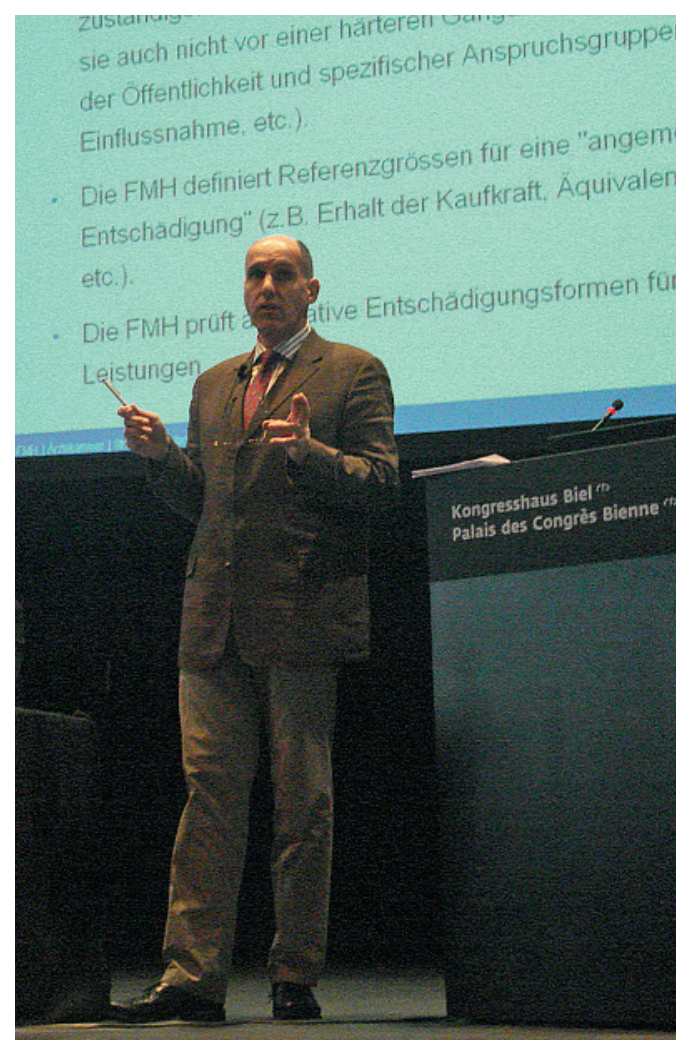

Le président Jacques de Haller lors d'une présentation.
Le président demande à la Chambre médicale d'approuver le document stratégique avec les modifications et compléments approuvés au cours de la discussion. D'ici la prochaine Chambre médicale, ce document servira de base au Comité central et à l'Assemblée des délégués pour leurs réflexions stratégiques. Il sera mis à jour en fonction de la situation politique, et si possible complété par les visions proposées, pour être à nouveau soumis aux délégués à la prochaine Chambre médicale ordinaire de mai 2009. Il est approuvé sous sa forme modifiée et complétée par une claire majorité des votants, avec une opposition et 3 abstentions.

\section{Budget 2009 de la FMH}

\section{Fixation de la contribution de base} et des contributions spéciales éventuelles

Pour ce point de l'ordre du jour, le président donne la parole au secrétaire général. Daniel Herzog commence par mentionner les documents qui ont été remis aux délégués concernant le budget 2009. Celui-ci boucle avec un déficit prévu d'env. 360000 francs. Lors de sa séance d'avril 2008, l'Assemblée des délégués a décidé que la contribution spéciale pour l'assistanat au cabinet médical devrait être intégrée dans la cotisation de base de la FMH. Le budget actuel comprend aussi une augmentation de la cotisation de base de 50 francs pour les nouveaux projets que la FMH a l'intention de lancer en 2009. Ceux-ci nécessitent la création de nouveaux postes de travail dans les domaines Données, démographie et qualité/ DDQ, eHealth, SwissDRG et TARMED, ainsi que dans les secteurs du Managed Care, du secrétariat de la FPPC et du secrétariat de direction. Des dépenses supplémentaires résultent également de la décision d'avoir une présidence séparée pour l'Assemblée des délégués et du personnel administratif nécessaire pour cet organe de la FMH. La création de nouveaux postes de travail et le nouveau décompte des cotisations AVS/AC sur les indemnités de séance et les honoraires induisent des charges supplémentaires au niveau des prestations sociales. En outre, les pertes de recettes résultant de la restitution des cotisations de membres restent importantes. Le budget ne contient d'ailleurs aucune réserve inutile, et ne prévoit pas non plus de nouvelles sources de recettes. Sans cette augmentation de la cotisation de 50 francs, le déficit s'élèverait à 1,5 million francs.

De surcroît, il convient également de tenir compte des mesures de consolidation du budget adoptées par la Chambre médicale en 2007. Elles portent en particulier sur une réduction des coûts du Comité central et sur l'optimisation du Secré- 
tariat général. Il s'agit là d'un mandat durable qui doit être pris au sérieux. Cela étant, lorsqu'on confie de nouvelles tâches à la FMH, il faut également lui procurer les ressources nécessaires.

Susanne Ernst/ASMAC observe que l'Assemblée des délégués a discuté en avril 2008 du budget 2009 et qu'elle l'a approuvé. Une majorité des délégués de l'ASMAC présents à la Chambre médicale expriment toutefois leur déception car, parmi les mesures de stabilisation adoptées par la Chambre médicale 2007, la seule qui a été appliquée concerne précisément la suppression de la restitution, après l'acquisition d'un titre de spécialiste, des cotisations versées par les médecins en formation postgraduée. L'ASMAC ne comprend pas non plus l'augmentation des coûts salariaux ni les projets coûteux tels que celui ayant trait à la Carte de professionnel de santé (CPS). Roland Schwarz/CdG relève que depuis l'AD d'avril 2008, d'autres mesures de stabilisation ont été mises en œuvre: la majeure partie du module prévoyant des mesures de rationalisation au Secrétariat général a p.ex. déjà été mise en œuvre. On travaille actuellement d'arrache-pied pour parvenir à une réduction des coûts au Comité central. Mais il ne s'agit pas d'une tâche facile quand les tâches confiées à la FMH sont élargies, comme il est ressorti de la présentation du budget par le secrétaire général. R. Schwarz précise en outre que la suppression de la rétrocession de cotisations décidée en décembre ne deviendra effective et donc perceptible qu'en 2013/2014. Il explique ensuite pourquoi la CdG a plaidé en faveur d'une augmentation de la cotisation de base: les nouveaux postes de travail sont des postes de dépenses réguliers qui devraient être compensés par des postes de recettes réguliers.

Philippe Luchsinger/ZH aimerait savoir ce que contient le compte «Salaires versés à des tiers» sous le poste Comité central, qui n'existait pas encore en 2008. Le secrétaire général explique qu'il ne sera désormais plus possible de verser des indemnités aux délégués sans procéder à un décompte de cotisations AVS. Ces indemnités figuraient autrefois sous le compte 4400/Commis- sions et délégations mais elles ont dues être transférées dans le compte «Salaires versés à des tiers» (cf. document «Remarques concernant le budget 2009»). Philip Dreiding/ZH fait part de son étonnement à propos des coûts du service juridique de la FMH qui ont augmenté d'un tiers. HP. Kuhn/ responsable du service juridique et secrétaire général adjoint explique que le bureau d'expertises extrajudiciaires est désormais inclus dans le budget du service juridique. Lors du regroupement des deux anciens Bureaux (Lausanne et Berne), on a passé du principe du mandat à celui de l'engagement salarial, d'où cette augmentation, bien que le Bureau d'expertises extrajudiciaires actuel compte moins de collaborateurs qu'autrefois.

La parole n'étant plus demandée, le président soumet tout d'abord au vote la proposition du Comité central $\mathrm{n}^{\circ} 1$ relative au point 3 concernant l'approbation du budget 2009 avec les cotisations de base indiquées. La Chambre médicale approuve le budget 2009 de la FMH et les cotisations de base proposées (tab. 1) par 98 voix contre 39 et 9 abstentions. (Remarque de la rédactrice du procèsverbal: la contribution spéciale CPS de 25 francs, adoptée par la Chambre médicale en 2006 pour 3 ans - 2007 à 2009 - figure dans le budget 2009.)

\section{Contribution spéciale NAKO (Newlndex)}

Le président donne la parole à Ernst Gähler, viceprésident et responsable CC du domaine Tarifs et conventions pour présenter la proposition du Comité central $\mathrm{n}^{\circ} 2$ relative au point 3 concernant la contribution spéciale NAKO (Service national de consolidation /NewIndex).

E. Gähler demande aux délégués d'approuver cette contribution car les chiffres fournis par le NAKO sont très importants aussi bien pour la FMH que pour les sociétés cantonales de médecine et les sociétés de discipline médicale dans le cadre des négociations tarifaires, des adaptations de la valeur du point tarifaire et des révisions de chapitres du TARMED. Sans ces chiffres, nous serions totalement livrés à l'arbitraire des assureurs. Jean-Pierre Pavillon/VD ne conteste pas la contribution versée par la FMH au NAKO mais

Tableau 1

Cotisations de base des membres pour 2009.

Catégorie de cotisation

1

2

3

4

5

6
Désignation

Médecins en pratique privée

Médecins salariés exerçant une fonction dirigeante

Médecins salariés exerçant une fonction non dirigeante

Médecins en formation postgraduée en vue d'un titre fédéral de spécialiste

Médecins domiciliés et exerçant à l'étranger

Membres temporairement sans activité médicale
Montant Fr.

660.00

660.00

330.00

330.00

165.00

165.00 
aimerait savoir, compte tenu des nombreuses sources de financement alimentant NewIndex, quels montants sont versés et pour quelle affectation. Pour le savoir, il aimerait mandater la FMH pour requérir les documents comptables de NewIndex. J. de Haller répond que toutes les sociétés cantonales de médecine qui décident de collaborer avec les centres fiduciaires sont les bienvenues, y compris la Société vaudoise de médecine. Les actionnaires de NewIndex disposent de tous les chiffres comptables. Mais pour la FMH, il n'est guère possible d'exiger d'une société par actions externe et indépendante de lui livrer ses chiffres. Les délégués de la FMH au conseil d'administration nous fournissent les informations nécessaires, de sorte que nous pouvons être sûrs que cet argent est dépensé de manière judicieuse. A. Haefeli/AG ajoute en sa qualité de président du conseil d'administration de NewIndex que les comptes annuels ainsi que tous les rapports annuels de NewIndex sont postés sur le site internet www.newindex.ch où ils peuvent être consultés librement.

Gabriele Merki/ASMAC demande pourquoi les contributions versées à NewIndex ne sont pas perçues avec les cotisations cantonales et elle constate que cette accumulation de contributions spéciales devient une charge financière toujours plus lourde, en particulier pour les médecins travaillant à temps partiel. De ce fait, elle demande que cette contribution soit divisée par moitié pour les médecins travaillant à $50 \%$ ou moins. Le président ne trouve pas que les contributions spéciales soient nombreuses car elles ont pratiquement toutes été supprimées. Il attire l'attention sur le fait que les sociétés de discipline médicale ont aussi besoin des statistiques de NewIndex pour leur activité, raison pour laquelle cette contribution ne peut pas être perçue par les sociétés cantonales de médecine. Le président demande ensuite à $A$. Haefeli/AG de répondre à l'autre question. Celui-ci explique que la contribution NAKO correspond en fait à une part importante de la caisse de NewIndex. La contribution NAKO ne concerne toutefois pas seulement le domaine de la LAMal, mais aussi celui des assurances nationales, la LAA et les adaptations de la structure tarifaire. Ces dernières sont très clairement une tâche de la FMH. Quant aux sociétés cantonales de médecine, elles financent leur part, les négociations de la valeur du point tarifaire, etc., par leur propre budget. E. Gähler souligne que sans les chiffres NAKO, la FMH ne parviendrait à rien dans les négociations sur la valeur du point tarifaire avec la SUVA et que le service tarifaire a aussi besoin de ces chiffres pour conseiller les sociétés de discipline médicale. Il précise en ou- tre que seules les catégories de cotisations 1 et 2 sont concernées par cette contribution spéciale. Philippe Rheiner/GE indique que la transparence existe chez NewIndex. Le meilleur endroit pour discuter des chiffres et des faits est l'assemblée générale de cette dernière et non la Chambre médicale. Dans ce contexte, il est regrettable que les actionnaires soient si peu nombreux à participer à l'assemblée générale de NewIndex. Il appelle donc les sociétés cantonales de médecine à y envoyer absolument leurs délégués.

Le président invite la Chambre médicale à se prononcer tout d'abord sur la proposition de G. Merki/ASMAC («Les médecins qui travaillent moins de $50 \%$ ne doivent pas verser cette contribution spéciale.»). Celle-ci est rejetée à une claire majorité.

La Chambre médicale approuve ensuite, à une grande majorité et par quelques oppositions, la proposition $\mathrm{n}^{\circ} 2$ relative au point 3 concernant la contribution spéciale NAKO. Cette contribution est perçue exclusivement auprès des catégories de cotisation 1 («Médecins en pratique privée») et 2 («Médecins salariés exerçant une fonction dirigeante»).

Après l'approbation du budget 2009 incluant les cotisations de base et la contribution spéciale NAKO, le président pose à la Chambre une question supplémentaire.

Ainsi que le prévoient les statuts, le délai d'opposition de 60 jours contre les décisions prises aujourd'hui courra dès la publication du procès-verbal de la séance de ce jour en français, allemand et italien dans le Bulletin des médecins suisses. Ce procès-verbal sera probablement publié à fin janvier 2009. Les décisions de la Chambre médicale extraordinaire de ce jour n'entreront donc en vigueur qu'à fin mars 2009. Certaines organisations de base craignent d'avoir des problèmes organisationnels. En d'autres termes, elles ne pourront envoyer les factures de cotisation à leurs membres qu'autour de Pâques. La question posée aux délégués est donc la suivante: acceptent-ils de déclarer urgentes les deux décisions relatives au budget avec les cotisations de base et à la contribution spéciale NAKO? Conformément aux statuts, le délai référendaire disparaîtrait pour ces deux décisions qui entreraient ainsi immédiatement en vigueur. Les organisations de base pourraient alors envoyer leurs factures à la date habituelle. La déclaration d'urgence d'une décision nécessite la majorité de ${ }^{4} / 5$ des voix. Pour CharlesA. Favrod-Coune/SMSR, une décision d'urgence est toujours aussi une «limitation des droits populaires». Nos membres pourraient estimer que l'on bafoue leurs droits. Il ne peut d'ailleurs pas s'imaginer que quelqu'un fasse opposition 
au budget adopté ce jour. C'est pourquoi il se prononce contre cette proposition parce que l'urgence n'est concrètement pas donnée. Personne d'autre ne demande la parole. Le président invite les délégués à voter. Les voix pour et contre sont à peu près en équilibre. Par conséquent, un comptage précis des voix n'est pas nécessaire car la majorité des quatre-cinquième n'a pas pu être atteinte.

\section{Révision partielle de la LAMal}

\subsection{Proposition J. Schlup/BE: Modèle} Forster/Oggier (assurance-maladie duale)

Jürg Schlup/Berne présente sa proposition qui, sous le point 1, demande l'avis et les propositions de la FMH au sujet du modèle Forster/Oggier et, sous le point 2, une décision de la Chambre médicale relative à un référendum pour le cas où les Chambres fédérales approuveraient le projet de révision LAMal élaboré par Forster/Oggier. Lorsqu'elle a rédigé sa proposition, la société de médecine du Canton de Berne pensait que le modèle Forster/ Oggier serait débattu au Conseil des Etats. Or, la Commission du Conseil des Etats (CSSS-E) a définitivement rejeté ce modèle il y a trois semaines en classant l'affaire. Le deuxième point de la proposition bernoise est donc, à son avis, devenu sans objet. D'un autre côté, les Chambres fédérales devront trouver une solution au cours des douze prochains mois et il est prévisible que des modèles semblables seront à nouveau proposés. Le président confirme que la CSSS-E a effectivement rejeté le modèle Forster/Oggier lors de sa dernière séance mais il rappelle que, durant toutes les séances qu'elle a tenues en 2008, elle a refusé un modèle et en a accepté un autre. Grâce à ses activités de lobbying et à ses contacts de plus en plus efficaces au sein du Parlement, la FMH a, conjointement avec la Conférences des directeurs de la santé (CDS), obtenu à cette séance de la CSSS-E qu'un nouveau débat soit lancé dans la bonne direction, ce qui est très positif. La position de la FMH concernant le modèle Forster/Oggier (cette question figure dans la proposition des Bernois) est la suivante: il y a environ deux ans et demi, la FMH a soumis au Conseil des Etats les thèses adoptées par la Chambre médicale de l'époque au sujet du Managed Care comme solution possible pour l'organisation des soins et des fournisseurs de prestations. Nos thèses étaient et restent un modèle dual avec le Managed Care comme « $2^{\mathrm{e}}$ voie», ce qui serait une bonne solution; la $2^{\mathrm{e}}$ voie suggérée par Forster/Oggier, qui vise la liberté de contracter et un marché totalement dérégulé, est en revanche inacceptable.
Le président précise que ce débat comprend deux niveaux qu'il faut séparer très clairement, à savoir la planification des soins et la démographie médicale, d'une part, et l'organisation de l'offre des prestations d'autre part. Le projet commun FMH/CDS concerne le premier niveau (régulation en fonction des besoins, soit par la limitation des autorisations d'exercer, soit surtout par la promotion de la relève médicale dans les cantons). Ce projet qui a finalement été rejeté en août par le Conseil des Etats sera probablement repris. Si le Conseil des Etats ne le fait pas, c'est le Conseil national qui le fera par la voie d'une initiative parlementaire. Pour le deuxième niveau, nous demandons, comme mentionné dans nos thèses sur le Managed Care, que le libre choix soit laissé à tous, aux spécialistes comme aux médecins de premier recours, aux adeptes du Managed Care comme à ses opposants, mais aussi aux patients. Selon l'issue des débats menés durant la session d'hiver au Conseil des Etats, le tout sera transmis au Conseil national ou reviendra à la Commission du Conseil des Etats.

Revenant à la proposition de J. Schlup/BE, le président plaide également en faveur du maintien du deuxième point. Nous signalerons ainsi clairement que la situation est inacceptable pour la FMH et cela nous donnera le levier politique dont nous aurons besoin ces douze prochains mois. Cette proposition recueille l'approbation de l'assistance. H. Chaudhry/ASMAC aimerait une explication de la part du président au sujet des conséquences financières qui affecteront les patients ne se ralliant pas au Managed Care, problématique évoquée dans les thèses de la FMH sur ce sujet. Le président explique que la FMH est elle aussi en faveur d'incitations financières, mais avec une différence tout à fait essentielle par rapport au modèle de santésuisse. Ce dernier prévoit que les assurés qui veulent conserver leur modèle actuel devraient payer le double. Celles et ceux qui optent pour le Managed Care paieraient la même prime qu'aujourd'hui. En revanche, la FMH propose que les assurés choisissant le Managed Care paient moins et que ceux qui veulent conserver leur modèle actuel s'acquittent de la même prime qu'aujourd'hui. François-Gérard Héritier/SSMG souligne que la Société suisse de médecine générale (SSMG) n'approuverait pas un référendum, parce qu'elle estime que le modèle Forster/Oggier ne va pas dans une direction si différente de ce que la FMH souhaiterait. Il faudrait, bien entendu, encore corriger certains points de ce modèle. Le président répond qu'il ne s'agit pas aujourd'hui de décider de lancer un référendum. D'ailleurs, on sait que la SSMG a écrit au Conseil 
des Etats pour lui dire qu'elle pourrait approuver le modèle Forster/Oggier sous réserve de quelques nuances, ce qui a occasionné quelques difficultés à la FMH. Il est d'avis que la SSMG n'a pas communiqué de manière habile, car le modèle en question contient de très nombreux dangers. Franziska Zogg/ZG observe que l'on ne connaît pas du tout les détails du modèle Forster/Oggier. On court le risque d'être vus comme des «Neinsager» lorsqu'on s'oppose à quelque chose que l'on ne connaît pas du tout. Elle aimerait que l'on prenne fait et cause pour une amélioration de la compensation des risques, qui devrait être mesurée d'après des critères liés à la morbidité. U. Stoffel/ $\mathrm{ZH}$ soutient cette intervention en ce qui concerne la compensation des risques. Le développement de cette dernière s'est arrêté à mi-chemin, ce qui revient à pénaliser les soins périphériques. Le modèle Forster/Oggier est le seul à contenir une compensation des risques axée sur la morbidité. Une compensation de ce type devrait être une condition sine qua non pour la FMH. Quelques délégués se rallient à cette proposition et mettent simultanément en garde contre le fait de juger positivement le modèle Forster/Oggier uniquement parce qu'il prévoit une compensation des risques orientée sur la morbidité. Ignazio Cassis/ vice-président informe que le débat au Parlement est fortement polarisé. On y oppose le «libre marché» (prôné par le modèle Forster/Oggier et qui n'est momentanément plus à l'ordre du jour du Parlement) et l'«étatisation» (suggérée par le modèle FMH/CDS et plutôt soutenue par la gauche). Il met en garde contre des signaux politiques forts s'opposant à quelque chose d'encore trop flou et qui n'est d'ailleurs actuellement plus un sujet de discussion. Il souhaite que l'on approfondisse cette discussion à la Chambre médicale de mai, lorsque la situation sera plus claire à bien des égards.

Le président constate finalement qu'on ne peut pas escompter de décision claire aujourd'hui, et il souhaite par conséquent renoncer à un vote sur le point 2 de la proposition bernoise.

Il invite toutefois les délégués de la Société de médecine du Canton de Berne à élaborer, d'ici la fin de la pause de midi, une proposition pour une meilleure compensation des risques en tant que complément à la proposition $\mathrm{n}^{\mathrm{o}} 1$ relative au point 4.1 .

La séance est interrompue à $12 \mathrm{~h} 36$ pour la pause de midi. La séance de l'après-midi débute à $13 \mathrm{~h} 35$ avec la poursuite de la discussion sur le point 4.1.

Le président constate que le $1^{\text {er }}$ point de la proposition bernoise a été débattu et que le deuxième point a été retiré.
Les sociétés de médecine des cantons de Berne, Genève, Schaffhouse, Lucerne, Soleure, Uri, Zurich, St-Gall, Obwald/Nidwald, Argovie, Thurgovie, Schwyz, Appenzell, Grisons, Bâle-Ville et Zoug proposent la formulation suivante pour un nouveau point 3 dans cette proposition: «La FMH soutient uniquement les modèles qui incluent une compensation des risques fondée sur la morbidité et dont la base de coûts se réfere à des coûts économiques globaux.»

U. Stoffel/ZH et C. Ramstein/VEDAG proposent une solution alternative: "La FMH estime que la compensation des risques fondée sur la morbidité doit impérativement figurer dans tout modèle.»

Lors du vote qui suit, la proposition des présidents cantonaux est acceptée en tant que $3^{\mathrm{e}}$ point de la proposition 4.1. à une grande majorité, sans opposition et avec quelques abstentions.

\subsection{Proposition Maurice Stauffacher/SVM}

Le contenu de cette proposition (obligation de contracter) vient d'être discuté de manière détaillée sous le point 2 et a été intégré dans le document stratégique de la FMH. Comme son auteur estime qu'il n'est pas nécessaire de la réexaminer ici, il la retire, ce qui entraîne simultanément l'annulation de la proposition de modification relative à la proposition 4.2 (M. Stauffacher/SVM), présentée en cours de séance par $H$. Chaudhry/ ASMAC (et également prise en considération dans le document stratégique).

\subsection{Proposition Markus Reber/LU}

Markus Reber/LU, invité par le président à expliquer sa proposition, se réfère à la proposition émise dans la prise de position «Régulation des ressources et promotion du Managed Care» de la FMH du 27 juin 2008, proposition visant l'introduction d'un nouvel alinéa 5 dans l'art. 12 de la LAMal: «Les caisses-maladie ne gèrent pas d'institutions de soins médicaux pour leurs assurés et ne participent pas financièrement à de telles institutions.» Il complète cette demande par la phrase suivante: «A l'inverse, les fournisseurs de prestations ne participent pas financièrement à des assurances.» La discussion animée qui s'ensuit révèle des avis très divergents. Quelques délégués indiquent que les cantons sont également des prestataires de services et qu'aux Grisons, certains cabinets médicaux ne survivent que parce qu'ils sont financés par les caisses-maladie et que l'on ne doit pas mettre en péril la dispensation directe de médicaments. HP. Kuhn constate que cette dispensation est en tous les cas une prestation médicale et qu'elle n'est certainement pas l'affaire des assureurs. J. de Haller retient de la discussion que le texte proposé est ressenti par la majorité des délégués comme étant 
trop peu clairement formulé. Il propose à Markus Reber de confier son texte au Comité central comme fil conducteur pour sa mise au point, et donc de ne pas voter maintenant sur sa proposition. M. Reber/LU, qui n'aimerait pas modifier sa proposition, approuve finalement la suggestion du président après que celui-ci l'a assuré que le Comité central rédigerait une proposition claire d'ici la Chambre médicale de mai. Il demande que cela figure au procès-verbal.

\section{Renforcement et autonomisation de la CFPC}

Max Giger/président de la CFPC rappelle aux délégués présents la situation initiale et l'évolution de ce projet jusqu'à la séance de ce jour. La loi sur les professions médicales (LPMéd), une motion de la Conseillère nationale $\mathrm{B}$. Heim et les exigences du Conseil suisse de la science et de la technologie (CSST) ont agi comme déclencheurs. Un groupe de travail a ensuite été chargé d'élaborer différentes propositions pour contrer ces exigences. Enfin, la Chambre médicale de mai 2008 a adopté dans le cadre d'un vote consultatif (en l'absence d'un quorum due à l'heure avancée) le modèle «CFPC light» élaboré par ce groupe de travail. Ce modèle prévoit une autonomisation juridique de la Commission au sein de la FMH. On veut ainsi essentiellement adopter un statut autonome et indépendant vis-à-vis de l'extérieur, parvenir à un renforcement et à une consolidation à l'intérieur et s'assurer une certaine souplesse pour pouvoir réagir rapidement aux changements qui surviennent au niveau politique. Une modification des statuts et du règlement d'exécution a été élaborée en vue de concrétiser le modèle adopté.

Les adaptations des statuts et du règlement d'exécution soumises à la Chambre dans la proposition $n^{0} 6.1$ portent essentiellement sur une nouvelle présentation et un nouveau nom: il est prévu que la CFPC actuelle s'appellera l'Institut suisse pour la formation postgraduée et continue des médecins (IFPC). Les compétences en matière de formation postgraduée et continue et la compétence financière dans le cadre du budget de la FMH seront transférées à cet Institut. Le droit à la signature sera réglé dans le Règlement de l'Institut. Il devrait être possible d'admettre d'autres organisations en qualité de membres ayant droit de vote (p.ex. CDS, H+) et le président de l'Institut devrait être élu à l'avenir par la Chambre médicale (il ne s'agira plus obligatoirement d'un membre du CC, ce qui assouplira le profil d'exigences et augmentera le nombre de candidats potentiels). Dans la discussion, les délégués critiquent avant tout l'abréviation choisie pour le nom de cet Institut. Invités à choisir entre l'abré- viation «IFPC» et «ISFPC», la majorité des délégués opte finalement pour l'abréviation «ISFPC», soit «Institut suisse pour la formation postgraduée et continue». M. Müller/ CMPR + SSMG, qui était pour une autonomisation plus large de la CFPC, remercie le groupe de travail et ses accompagnants le Dr M. Giger (actuellement président de la CFPC) et Christoph Hänggeli (administrateur responsable de la FPPC) pour le travail accompli en faveur de ce bon projet porteur d'avenir.

\section{Modifications des statuts et du règlement d'exécution}

\subsection{Révision des statuts et du règlement} d'exécution: renforcement et autonomisation de la CFPC

Le président invite les délégués à voter sur la proposition $n^{\circ} 1$ de la CFPC et du Comité central relative au point 6.1. La Chambre médicale approuve par 149 voix sans opposition et avec 2 abstentions les modifications des statuts et du règlement d'exécution visant à renforcer et à autonomiser la CFPC, y compris l'abréviation «ISFPC» adoptée au point 5 pour l'Institut suisse pour la formation postgraduée et continue.

6.2 Mise à jour des statuts et du règlement d'exécution: modèles de direction, présidence de I'AD et diagramme révisé du processus de planification et de budgétisation

Cette affaire n'a pas non plus été traitée à la Chambre médicale ordinaire de mai 2008 par manque de temps.

M. Kondo Oestreicher/SSPTC demande que l'on écrive correctement le nom de sa société de discipline médicale dans l'annexe II des statuts de la FMH, aussi bien dans la version française qu'allemande (Société suisse de pharmacologie et toxicologie clinique).

Roland Oppliger/SSAI attire l'attention sur le fait qu'il faut remplacer l'abréviation CFPC par ISFPC à l'art. 22, let. $3^{\text {bis }}$, du règlement d'exécution (RE). Thomas Keller/SZ émet une suggestion concernant les statuts: les secrétaires généraux ou les directeurs des organisations de base et de discipline médicale peuvent aussi participer aux séances de la Chambre médicale (art. 9, let. 6 du RE). Les petites sociétés cantonales comme la sienne n'ont pas de directeur ou de secrétaire général pour des raisons financières mais il devrait aussi leur être permis, le cas échéant, de s'adjoindre un conseiller lorsqu'elles viennent à la Chambre médicale. J. de Haller en prend note pour la prochaine révision des statuts et du règlement d'exécution en vue de l'élaboration d'un texte approprié. 


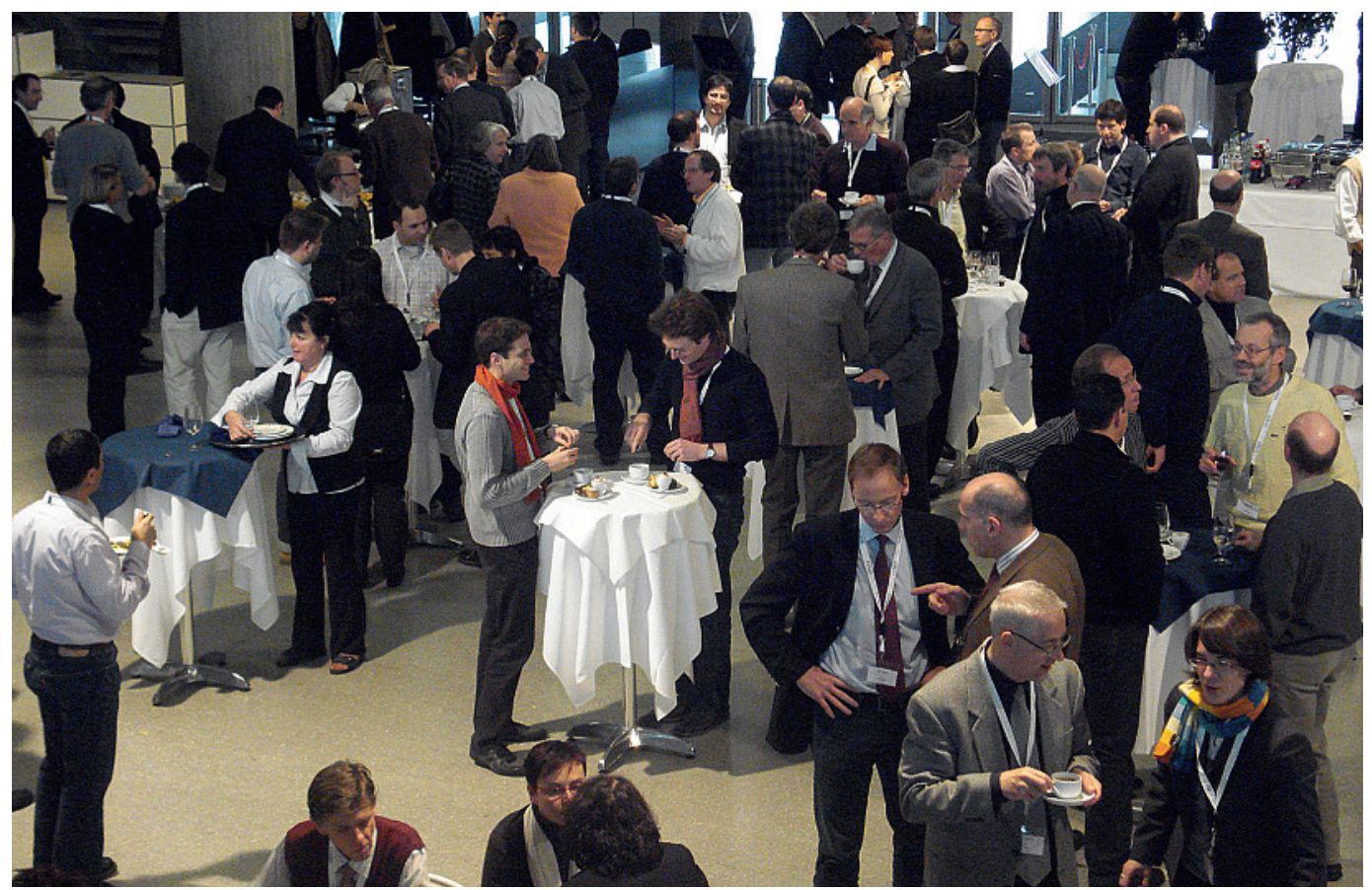

Conversations animées pendant la pause de midi.

Personne d'autre ne demandant la parole, le président soumet au vote la proposition $n^{0} 1 \mathrm{du}$ Comité central relative au point 6.2. La Chambre médicale approuve les modifications des statuts et du règlement d'exécution telles que présentées par 155 voix sans opposition ni abstention.

\subsection{Inclusion de la Société suisse de chirurgie de la main et de la Société suisse de} neuropathologie dans I'annexe II des statuts La parole n'est pas demandée. La proposition $n^{0} 1$ de la CFPC et du Comité central relative au point 6.3 est approuvée par la Chambre médicale sans opposition ni abstention. Par conséquent, ces deux sociétés seront incluses dans l'annexe II «Sociétés de discipline médicale reconnues», conformément à l'art. 18 des statuts de la FMH.

\subsection{Proposition C. Ramstein/VEDAG, point 6.4}

Invitée à s'exprimer par le président, la Chambre médicale décide tout d'abord à une grande majorité et avec quelques oppositions et abstentions (la majorité des deux tiers est donc atteinte) d'entrer en matière sur cette proposition. C. Ramstein/ $V E D A G$ motive sa demande visant à ce qu'à l'avenir, les affaires statutaires (il entend par là notamment le budget, les cotisations de membres et les contributions spéciales) figurent non seulement à l'ordre du jour de la Chambre médicale ordinaire mais y soient aussi traitées. Si le budget de la FMH n'est pas adopté à la Chambre médicale ordinaire organisée dans le courant du $2^{\mathrm{e}}$ trimestre, cela crée des problèmes pour les sociétés can- tonales de médecine. Leurs assemblées de membres ou de délégués, tenues soit au cours du $1^{\mathrm{er}}$ semestre soit au plus tard en novembre de l'année civile, doivent voter sur leurs propres budgets et cotisations. Leur budget est planifié sur la base $\mathrm{du}$ budget et des cotisations et contributions spéciales de la FMH approuvés par la Chambre médicale. J. de Haller comprend certes cette demande, mais il souhaite conserver une certaine souplesse et éviter une sur-réglementation. HP. Kuhn/secrétaire général adjoint précise que l'on peut faire usage des motions d'ordre pour demander, entre autres, qu'une affaire figurant à l'ordre du jour soit traitée.

Le président soumet au vote la proposition de C. Ramstein/VEDAG. Les délégués la rejettent par 88 voix contre 47 , sans abstention.

\section{Confirmations pour l'Assemblée des délégués de la FMH}

Le président demande à la Chambre médicale de confirmer en bloc les nouveaux délégués ordinaires et délégués suppléants pour l'Assemblée des délégués de la FMH:

Délégués ordinaires: Jürg Unger, Zurich/FMPP, Walter Kaiser, Thalwil/SFSM , Guillaume Favre, Genf/ASMAC; délégués remplaçants: Gerhard Schilling, Stein am Rhein/CMPR, Rolf Zundel, Bätterkinden/VEDAG, Lars H. Frauchiger, Ittigen/ASMAC.

La Chambre médicale confirme ces délégués ordinaires et délégués suppléants dans leur fonction sans opposition ni abstention. 


\section{Propositions pour décision et informations en provenance des domaines d'activité}

\section{Formation médicale}

\subsection{Création de titres, formations approfondies et attestations de formation complémentaire}

\subsubsection{Création de la formation approfondie Pédiatrie du développement}

M. Giger/CC+président de la CFPC explique cette proposition: la pédiatrie du développement est un domaine central de la pédiatrie. Des compétences spéciales sont nécessaires pour pouvoir investiguer les troubles complexes du développement et du comportement. La rédaction finale et la mise en vigueur de ce programme seront assumées par la CFPC. M. Giger souhaite la bienvenue à Jon Caflish, présent à titre d'invité pour répondre aux questions éventuelles. Les délégués ne demandent pas la parole.

Le président invite la Chambre médicale à se prononcer. Elle approuve la proposition $n^{\circ} 1$ de la CFPC et du CC relative au point 8.1.1 à une grande majorité, par 8 oppositions et 10 abstentions.

\subsubsection{Création de la formation approfondie} Psychiatrie de consultation et de liaison M. Giger/CC+président de la CFPC explique cette proposition: la psychiatrie de consultation et de liaison est un domaine autonome de la psychiatrie. La formation approfondie vise à transmettre des compétences particulières dans les soins médicaux de base pour les secteurs de la psychiatrie d'urgence et de la psychosomatique. La rédaction finale et la mise en vigueur seront assumées par la CFPC. M. Giger souhaite la bienvenue à Franz Caduff et Ulrich Schnyder, présents en qualité d'invités pour répondre aux questions éventuelles. La parole n'est pas demandée. Le président invite la Chambre médicale à voter. Elle accepte la proposition $n^{\circ} 1$ de la CFPC et du Comité central relative au point 8.1.2 par 78 voix, 52 oppositions et 17 abstentions.

\subsubsection{Création de l'attestation de formation} complémentaire Médecine d'urgence hospitalière M. Giger/CC+président de la CFPC rappelle tout d'abord qu'une première lecture de ce document a déjà eu lieu à la Chambre médicale extraordinaire du 6 décembre 2007. La CFPC a approuvé cette attestation en septembre et le Comité central en octobre. La Société suisse de neurologie et la Société suisse d'orthopédie et de traumatologie restent opposées à sa création. Comme pour les autres attestations, la rédaction finale et la mise en œuvre du programme seront assumées par la CFPC.

M. Giger souhaite la bienvenue à Lion Bernoulli et Ulrich Bürgi, délégués de la SSMUS, qui se tiennent à disposition pour répondre aux questions éventuelles. M. Dutoit/SSO confirme une fois de plus qu'il a toujours été opposé à la création de nouvelles formations approfondies et attestations de formation complémentaire. Dans le cas concret, il est clairement convaincu qu'on pourrait régler les cas d'urgence sur le plan régional et non au niveau suisse. La nécessité d'une telle attestation reste selon lui à prouver. M. Giger répond qu'il ne s'agit ni d'édicter une réglementation nationale ni de prescrire des dispositions sur le plan local ou régional.

T. Keller/SZ demande comment les pays européens règlent cette question. U. Bürgi/SSMUS informe qu'en Europe, pas moins de 13 pays connaissent déjà un titre de spécialiste en médecine d'urgence. Actuellement, la Roumanie et l'Albanie sont en train de créer un tel titre; la Suisse est donc l'un des derniers pays où la médecine d'urgence ne bénéficie pas encore d'une formation structurée. Hans-Ueli Würsten/AMDHS confirme que ce type de formation existe dans toute l'Europe. Si nous ne réglons pas maintenant ce point, nous devrons importer des urgentistes d'Europe. Brigitte Muff/ZH explique que personne n'a pu jusqu'à présent la convaincre personnellement d'obtenir cette attestation de formation complémentaire. Elle comprend encore moins pourquoi celle-ci devrait être rattachée à une association composée essentiellement d'anesthésistes et d'internistes. U. Bürgi/SSMUS répond qu'il appartient aux jeunes médecins de nous dire ce dont ils auront besoin à l'avenir. Le programme relatif à la médecine d'urgence hospitalière a été publié en 2005. Entre-temps, 350 médecins dont aussi des chirurgiens, l'ont suivi. Il regrette qu'au contraire d'autres pays, les chirurgiens suisses se soient trop peu souciés de la médecine d'urgence jusqu'à présent, ce qui est un désavantage pour les jeunes médecins.

Verena Briner/SSMI relève que la Société suisse de médecine interne soutient officiellement cette proposition. La SSMI est d'avis qu'on pourra ainsi atteindre une certaine standardisation et, partant, un bon niveau de qualité dans les différents services d'urgence. Le président soumet finalement la proposition au vote. La Chambre médicale approuve la proposition $n^{\circ} 1$ de la CFPC et du CC relative au point 8.1 .3 par 113 voix contre 29 et 12 abstentions. 


\section{Tarifs et conventions}

\section{Révision de la Liste des analyses}

Ernst Gähler/vice-président et responsable du domaine esquisse tout d'abord un bref historique de la révision de la Liste des analyses (LA). Suite à l'audition du 9 juillet 2008, le groupe de travail Laboratoire, mandaté par l'Assemblée des délégués et constitué de façon très représentative, a établi son propre calcul pour les analyses de laboratoire au cabinet du praticien selon des critères d'économie d'entreprise et montré clairement que la taxe de présence figurant dans la version Beta de l'OFSP ne saurait représenter une solution. Dans l'intervalle, l'OFSP a élaboré une version Gamma 02, laquelle a été terminée en avant même la fin de la consultation sur la version Beta 8.0 - l'opinion de la FMH n'a donc pas pu être prise en compte dans la version Gamma 02. Plusieurs positions de cette version sont encore moins bonnes que dans la version Beta 08. Le modèle «Point of Care» élaboré par le groupe de travail propose une tarification séparée de toutes les prestations de laboratoire pouvant être effectuées le jour même. Il s'agit d'une solution neutre quant aux coûts, c'est-à dire qu'elle n'entraîne ni économies ni coûts supplémentaires. Le 31 octobre 2008, la FMH a pu soumettre à l'OFSP son modèle «Point of Care» et lui expliquer pourquoi la taxe de présence n'était pas une solution. L'infrastructure des laboratoires de praticien n'est pas la même dans tous les cabinets et il n'existe pas de distribution normale du nombre d'analyses par séance. La version Gamma 03, valable actuellement, a été discutée lors de la séance de novembre de la Commission fédérale des analyses, moyens et appareils (CFAMA). Les propositions de la FMH sous la forme du modèle «Point of Care» ont été ignorées. La taxe de présence est maintenue. Deux problèmes se posent avec l'OFSP: d'une part le conseiller fédéral Couchepin et l'Office continuent à vouloir nous faire croire que le prix des analyses en Suisse est nettement plus élevé qu'en Allemagne, alors que les écarts ne se situent que dans une marge de -2 à $+2 \%$. D'autre part, l'Office a toujours refusé de nous indiquer ses bases de calcul. Le calendrier prévu se présente comme suit: l'OFSP remaniera le modèle en tenant compte des recommandations de la CFAMA et soumettra une proposition au Prof. Zeltner. Il s'ensuivra une consultation des offices, sur la base de laquelle le directeur de l'OFSP présentera une proposition au conseiller fédéral Pascal Couchepin. La décision de ce dernier est attendue pour janvier/février 2009, mais sa mise en vigueur n'aura pas lieu avant le milieu de l'année 2009.
Le groupe de travail Laboratoire de la FMH a élaboré un catalogue de mesures approuvé par l'Assemblée des délégués et par le Comité central. Des mesures sont prévues à différents niveaux: technique (nouvelle discussion avec le directeur de l'OFSP concernant le modèle de la FMH; négociations du point tarifaire avec santésuisse), politique (discussions avec des parlementaires, recherche d'alliances), médiatique (groupe de communication Laboratoire du praticien), mesures au niveau de la base (poursuite de la campagne de lettres et préparation de manifestations publiques).

La discussion qui s'ensuit le montre clairement: la colère sur la manière dont cette révision de la LA a été menée par Berne et dont on compromet ainsi l'existence des laboratoires de cabinet est grande, et les propositions de mesures qui en résultent sont très radicales. L'OFSP se rend peu crédible, notamment parce qu'il ne veut pas publier ses calculs sous prétexte qu'il s'agit de «travaux en cours». Cela prête à penser que tout n'est pas correct dans ces calculs. Un soupçon que confirme une comparaison de différents prix d'analyses entre les versions Beta et Gamma. Le groupe de travail Laboratoire tiendra compte de toutes les suggestions et remarques des délégués dans la suite de ses travaux.

\section{Réduction de la marge de distribution (dispensation directe de médicaments)}

Là aussi, il s'agit d'un chantier de longue date. En 2007, santésuisse et Helsana ont exigé une baisse des marges de distribution des pharmacies d'officine et de cabinet en arguant du fait qu'elles étaient trop élevées. En 2008, cette demande a été appuyée par le Surveillant des prix qui a exigé une baisse non pas de $3 \%$ mais de 7 à $8 \%$. Le Surveillant a néanmoins émis un avis favorable sur les pharmacies de cabinet et la dispensation directe de médicaments. Dans ce cas également, les bases de calcul n'ont jamais été présentées. Le conseiller fédéral $\mathrm{P}$. Couchepin a répondu à une lettre de la FMH en indiquant que les coupes auraient pu êtres plus importantes mais que, par égard pour la médecine de premier recours, l'on s'était contenté d'une baisse de $3 \%$ pour aboutir à une marge de $12 \%$. L'OFSP ordonnera cette baisse. Celle-ci n'aura pas lieu au $1^{\text {er }}$ janvier, mais dans le courant de l'année 2009. Elle touchera à nouveau principalement les médecins de premier recours dans les petits cantons, qui doivent déjà faire face à des valeurs du point basses. Sur cette question également, un groupe de travail largement représentatif élabore des solutions. Un modèle proposé par la SSMG est actuellement à l'étude. Personne ne demande la parole. 
Négociations de la valeur du point tarifaire dans le domaine LAA

Selon E. Gähler, il s'agit là d'une autre histoire sans fin qui a débuté il y a plus d'un an. En mai, les délégations aux négociations de la FMH et de la CTM se sont mises d'accord sur une adaptation de la valeur de point de $+5 \%$, échange électronique des données inclus. Le conseil d'administration de la CTM a toutefois rejeté le résultat de ces négociations à la fin juin. Elles ont donc dû être suspendues. Finalement, le 18 novembre 2008, après de nombreuses tentatives de la FMH restées vaines, une discussion au sommet a pu avoir lieu entre cette dernière et la CTM. Le 4 décembre 2008, le conseil d'administration de la CTM a débattu du contenu de ladite discussion. La lettre du conseil d'administration que la FMH a reçue hier contient une offre qui, bien que sérieuse, est liée à certaines conditions qu'il conviendra d'évaluer de manière approfondie. En principe, on peut émettre un avis prudemment positif. Une augmentation de la valeur du point serait possible au 1er mars 2009. Pour le cas où nous ne pourrions pas approuver une telle proposition, une éventuelle réaction du corps médical est à l'étude. Cela étant, il ne faut pas oublier qu'en mai 2008, le Conseil fédéral a décidé de réunir les valeurs du point dans les domaines LAMal et LAA dans le cadre d'un «projet 2012». Personne ne souhaite prendre la parole à ce sujet.

\section{Projet TARMED Suisse 2010}

Ce projet, qui aurait dû permettre de reprendre les révisions de la structure et des chapitres du TARMED, était sur la bonne voie jusqu'à ce que santésuisse veuille introduire un «couloir de croissance des coûts» dans la structure du projet. Cela est inacceptable non seulement pour la FMH, mais aussi pour la CTM et H+. santésuisse a jusqu'en février pour se pencher une nouvelle fois sur la question. La version 1.06 du TARMED, prévue pour 2009, n'entrera en vigueur qu'au $1^{\text {er }}$ mars. Le Conseil fédéral n'a pas encore donné son accord mais cela ne saurait tarder. Dans cette version 1.06, les indemnités forfaitaires de dérangement en cas d'urgence ont été pratiquement ramenées à leur valeur initiale. A cela s'ajoutent les forfaits en cas de visite pressante. A ce propos, il est réjouissant de constater que santésuisse et la CTM considèrent que nos chiffres et bases de données (NAKO/NewIndex) sont plus fiables que leurs propres bases de calcul. C'est une première!

Pour terminer, E. Gähler remercie les participants pour leur soutien tout en adressant des remerciements particuliers au responsable et aux collaborateurs du Service tarifaire pour l'énorme travail accompli à l'arrière-plan.

\section{SwissDRG}

Le président donne ensuite la parole à Pierre-François Cuénoud/membre du CC et responsable du domaine SwissDRG pour des informations d'actualité sur le projet du même nom. En janvier, l'association SwissDRG a été transformée en société anonyme et le bureau Casemix (CMO) a été mis en service. Simon Hölzer, son directeur, a recruté le personnel nécessaire et organisé le bureau. En juin, des décisions importantes en matière de statistiques ont été prises. En juillet, la version 0.1 du premier groupeur SwissDRG a pu être publiée. Le même mois a été lancé un cycle de propositions qui s'est terminé en novembre. La FMH a soutenu les sociétés de discipline médicale et les organisations faîtières dans l'élaboration de leurs propositions en mettant à leur disposition des experts des DRG et des spécialistes du codage. Au total, 1630 propositions ont été déposées par la FMH. P.-F. Cuénoud remercie vivement les sociétés de discipline médicale et les organisations faîtières de leur précieuse collaboration. Il s'agit désormais de recenser les données de l'année précédente (2007) et des calculs doivent être effectués en vue de la publication de la version $0.2 \mathrm{du}$ groupeur. Une nouvelle version du groupeur paraîtra chaque année. L'introduction effective du système tarifaire SwissDRG dans toute la Suisse est fixée par la loi au $1^{\text {er }}$ janvier 2012 au plus tard. Le financement du Bureau Casemix est estimé à 3 millions de francs par an pour ces trois prochaines années. Comme les recettes provenant des suppléments sur les forfaits ne pourront être encaissées qu'avec l'introduction du système SwissDRG, les partenaires du projet assurent, sous forme de prêt, un préfinancement de 9 millions de francs pour les 3 prochaines années. La contribution de la FMH se monte à 250000 francs par an, proportionnellement à sa participation au capital-actions. Par mesure de précaution, ce prêt a été inscrit au budget en procédant à une régularisation comptable. Les chances que nous puissions le récupérer avec intérêts sont cependant bonnes. Il reste toute une série de problèmes à résoudre, par exemple la question de la recherche concomitante qui est largement traitée par les médias ces temps-ci. Dans le cadre de la révision de l'OAMal, la FMH s'était exprimée en faveur du financement de cette recherche au moyen d'un supplément perçu sur les forfaits et avait proposé d'en attribuer la responsabilité à la société SwissDRG. Dans l'intervalle, le Conseil fédéral a désigné l'OFSP comme l'organe compétent. Celui-ci devra toutefois travailler en étroite collaboration avec tous les protagonistes et assurer notamment un accompagnement scientifique. Dans ce projet, la FMH a obtenu entre autres une participation à 
l'actionnariat de SwissDRG (à hauteur de 8\%), un siège au sein du conseil d'administration, deux sièges dans le groupe de référence Médecine ainsi qu'un siège dans le groupe de référence Economie (ces groupes assistent les unités organisationnelles correspondantes du Bureau Casemix). En même temps, la FMH pratique une politique d'information offensive. Cependant, il lui reste encore beaucoup à faire: elle doit notamment renforcer ses alliances avec ses partenaires, lutter pour garder voix au chapitre et, enfin et surtout, mettre sur pied une équipe SwissDRG au sein du Secrétariat général.

\section{Données, démographie et qualité}

Le président donne la parole à Daniel Herren/membre du CC responsable du domaine DDQ pour des informations sur son secteur d'activité. Les objectifs du domaine pour 2008 étaient d'augmenter la visibilité de la qualité en tant qu'élément essentiel de l'activité médicale, d'instaurer une collaboration avec les sociétés de discipline médicale et les sociétés cantonales de médecine et de créer les conditions nécessaires à une reconnaissance de la démarche qualité des médecins par leurs partenaires. Le domaine bénéficie du soutien très actif du Groupe de travail Qualité de la FMH (GTQ-FMH). En ce qui concerne le positionnement de la FMH en matière de qualité, les enquêtes et discussions effectuées au sein du GTQ$\mathrm{FMH}$, du CC et de l'AD et les rencontres avec les responsables qualité ont montré clairement que la FMH devrait assumer un rôle plus actif, situé entre celui de «catalyseur de changement» et de «concepteur actif». L'une des priorités fixées pour 2008 consistait à instituer un réseau Qualité pour promouvoir les échanges au sein du corps médical. Deux rencontres très constructives ont permis de clarifier la conception des différents rôles et de promouvoir les discussions communes. Deux autres réunions sont prévues. En outre, on s'est efforcé de mettre des connaissances spécifiques à la disposition des membres. Les premiers descriptifs d'initiatives qualité issues de la pratique publiés dans le BMS seront suivis par une deuxième série de publications à la fin de cette année. Le GTQ a en outre élaboré des bases scientifiques qui serviront de fondement à la formulation des avis de la FMH. Une ligne d'assistance par messagerie électronique (agq@fmh.ch) a été mise sur pied pour répondre aux questions ayant trait à la qualité. La stratégie qualité de l'OFSP ne peut en revanche guère être qualifiée de succès. La FMH fait certes partie de l'équipe de projet élargie (avec entre autres $\mathrm{H}+$, la CDS et santésuisse) mais pour l'instant, une seule séance s'est tenue en août pour présenter les dix champs d'action qui servi- ront de base à l'élaboration de la stratégie de la Confédération en la matière. Il n'y a quasiment pas eu de discussions à ce sujet et depuis, le silence règne. L'OFSP a reçu le mandat d'élaborer des recommandations pour ladite stratégie; on s'attend à ce que son rapport soit présenté à Pascal Couchepin en janvier, et nous espérerons obtenir ultérieurement des informations à ce sujet. Le dialogue avec santésuisse s'est avéré légèrement plus constructif: grâce à un projet de monitorage de la qualité présenté à l'Assemblée des délégués et lors de la rencontre des responsables qualité, il apparaît que nous sommes sur la bonne voie.

Le projet ReMed semble devenir une histoire à succès. Le projet-pilote lancé en 2007 dans les cantons de Thurgovie et de Neuchâtel a permis de suivre 26 cas jusqu'à présent, et il semble bien établi et de mieux en mieux accepté. Il a donc été décidé de l'étendre quelque peu, sur une base régionale plutôt que cantonale comme c'était le cas jusqu'ici, et d'élaborer un plan de financement en vue de l'introduction, en 2011, du projet dans toute la Suisse. Davantage d'informations et des contacts sont disponibles par le biais de www.swiss-remed.ch, info@swiss-remed.ch et 0800 OREMED (0800 073633).

En ce qui concerne la démographie, les objectifs 2008 du domaine étaient les suivants: élaborer et mettre à disposition des bases valables sur les questions actuelles de démographie médicale et contribuer à la mise sur pied de bases utiles et nécessaires concernant les prestations ambulatoires et hospitalières. Des projets portant respectivement sur le monitorage des médecins hospitalier et des fournisseurs de prestations médicales sont en cours dans ce contexte, tous deux en collaboration avec l'Obsan. La révision de la statistique médicale est lancée, la phase pilote est terminée et le nouveau masque restructuré de saisie des données figure sur le portail internet $m y \mathrm{FMH}$. Le feedback reçu jusqu'à présent a été positif à quelques exceptions près. Des informations sur les premiers résultats obtenus seront régulièrement diffusées et la publication de la statistique médicale dans le BMS est prévue pour mars.

Pour terminer, $D$. Herren évoque brièvement les perspectives pour l'année à venir: il va de soi que les travaux amorcés seront poursuivis. Le processus de formulation des objectifs 2009, basé sur les projets en cours, s'est terminé hier lors de la séance d'un groupe de travail ad hoc.

\section{Promotion de la santé et prévention}

Christine Romann/membre du CC et responsable $d u$ domaine se limite, dans ses informations, à la nouvelle loi sur la prévention et la promotion de la santé. La FMH salue cette loi notamment pour 
les raisons suivantes: elle permet de combler les lacunes juridiques existantes en ce qui concerne les maladies psychiques et les maladies non transmissibles et de donner un ancrage légal à des principes importants de promotion de la santé, elle prend en compte la libre détermination personnelle et la diversité de la population dans la mise en œuvre des mesures prévues, elle crée une terminologie uniforme comportant une définition des notions utilisées et elle introduit des objectifs nationaux, une stratégie du Conseil fédéral et des comptes rendus en matière de santé en tant qu'instruments de régulation. Par ailleurs, cette nouvelle loi clarifie les rôles entre les différents acteurs: la Confédération se concentre sur le domaine stratégique, les cantons gardent leur responsabilité en matière de prévention et de promotion de la santé et veillent à la mise en œuvre des mesures. Les organisations privées jouent un rôle primordial dans l'application des mesures et dans l'élaboration d'objectifs nationaux. En qualité de protagoniste fort et de centre d'excellence pour l'ensemble de la Suisse, un Institut suisse de prévention et de promotion de la santé soutiendra les cantons et les tiers dans l'application des mesures et jettera les bases d'une culture de la prévention. La procédure de consultation relative à cette loi s'est achevée à la fin octobre. Le rapport sur ses résultats et sur la suite du processus est attendu pour février prochain. En 2009, la loi sera soumise au Parlement et son entrée en vigueur est prévue pour 2012.

\section{Divers}

Au nom de la Conférence des sociétés médicales de Suisse orientale, Peter Wiedersheim/SG a soumis une proposition 1 au point 12 de l'ordre du jour. Le président lui donne la parole. L'auteur de la proposition constate que le projet HPC accuse un retard de plusieurs mois et que le manque de transparence et d'une gestion claire des avantages visés a entraîné le dépôt, lors de l'Assemblée des délégués de juin 2008, d'une proposition de moratoire et d'une demande d'intervention de la Commission de gestion (CdG). Ces propositions ont toutes deux été approuvées par l'AD. Il souhaiterait donc obtenir des informations quant aux coûts du projet et savoir où en est sa réalisation, connaître les points concrets contestés par la CdG et les mesures que le CC pense prendre pour éviter que certaines lacunes dans la gestion des compétences et des processus ne perdurent, et enfin savoir si le rapport de la CdG sera présenté à la ChM. Monique Gauthey/membre du CC et responsable $d u$ domaine eHealth explique que le moratoire a donné lieu à de nombreuses discussions approfondies et que deux groupes de travail ont été formés: le groupe de travail HPC, qui traite des applications de la carte de professionnel de santé, et un groupe de travail eHealth. J. de Haller ajoute que les six mois de retard dans la réalisation du projet sont dus au fait que la votation du $1^{\text {er }}$ juin a nécessité une redistribution des ressources au sein du Secrétariat général. L'introduction de la carte est prévue pour mai 2009, avant la séance de la Chambre médicale. Pour répondre aux deux questions restantes, le président donne la parole au président de la Commission de gestion. $R$. Schwarz indique que la CdG a demandé des informations sur la décision prise concernant le contrat principal passé entre la FMH et Swisscom et qu'elle a fait parvenir une lettre au CC qui a été discutée conjointement le 4 décembre. Le rapport mentionné par P. Wiedersheim figurera dans le rapport annuel de la CdG sur l'exercice 2008 qui sera présenté à la Chambre médicale ordinaire de mai 2009. Voilà pour ce qui est des questions formelles. Quant au contenu du contrat principal sur la HPC, R. Schwarz n'a rien à en dire. Le président souligne encore une fois que le projet est en bonne voie et qu'actuellement aucun problème majeur ne se pose. $P$. Wiedersheim/SG remercie pour les explications données, mais n'est pas entièrement satisfait. Il relève que l'Assemblée des délégués avait décidé que la CdG procéderait à une évaluation de l'ensemble du projet et pas seulement à une évaluation des procédures juste avant la dernière Chambre médicale. Il demande donc que la CdG évalue le projet dans son intégralité. Le président confirme que c'était justement là le mandat de la CdG. Comme convenu, cette dernière présentera son rapport à la Chambre médicale ordinaire de mai 2009.

Après s'être assuré que plus personne ne demande la parole, le président remercie l'assistance de sa coopération et des discussions constructives et clôt la séance à 16h55 en souhaitant à toutes et à tous une bonne et heureuse année 2009. 


\begin{tabular}{|c|c|c|c|}
\hline \multicolumn{4}{|l|}{ Glossaire } \\
\hline AC & Assurance-chômage & LAMal & Loi fédérale sur \\
\hline AD & Assemblée des délégués & & I'assurance-maladie \\
\hline AMDHS & $\begin{array}{l}\text { Association des médecins diri- } \\
\text { geants d'hôpitaux de Suisse }\end{array}$ & NAKO & $\begin{array}{l}\text { Service national de } \\
\text { consolidation des données }\end{array}$ \\
\hline ASMAC & $\begin{array}{l}\text { Association suisse des médecins- } \\
\text { assistant(e)s et chefs de clinique }\end{array}$ & OAMal & $\begin{array}{l}\text { Ordonnance sur } \\
\text { I'assurance-maladie }\end{array}$ \\
\hline AVS & Assurance vieillesse et survivants & Obsan & Observatoire suisse de la santé \\
\hline BMS & Bulletin des médecins suisses & OFSP & Office fédéral de la \\
\hline CC & Comité central & & santé publique \\
\hline CdG & Commission de gestion & RE & Règlement d'exécution \\
\hline CDS & $\begin{array}{l}\text { Conférence suisse des } \\
\text { directrices et directeurs }\end{array}$ & SFSM & $\begin{array}{l}\text { Swiss Federation for Specialities } \\
\text { in Medicine }\end{array}$ \\
\hline & cantonaux de la santé & SSAI & Société suisse d'allergologie \\
\hline CFAMA & $\begin{array}{l}\text { Commission federale des } \\
\text { analyses, moyens et appareils }\end{array}$ & SSDV & $\begin{array}{l}\text { et d'immunologie } \\
\text { Société suisse de dermatologie }\end{array}$ \\
\hline CFPC & $\begin{array}{l}\text { Commission pour la formation } \\
\text { postgraduée et continue }\end{array}$ & SSMG & $\begin{array}{l}\text { et vénéréologie } \\
\text { Société suisse de médecine }\end{array}$ \\
\hline CMO & $\begin{array}{l}\text { Bureau Casemix } \\
\text { (Casemix Office) }\end{array}$ & SSMI & $\begin{array}{l}\text { générale } \\
\text { Société suisse de médecine }\end{array}$ \\
\hline CMPR & Collège de médecine de & & interne \\
\hline CPS & $\begin{array}{l}\text { premier recours } \\
\text { Carte de professionnel de santé }\end{array}$ & SSMUS & $\begin{array}{l}\text { Société suisse de médecine d'ur- } \\
\text { gence et de sauvetage }\end{array}$ \\
\hline CSSS-E & $\begin{array}{l}\text { Commission de la sécurité } \\
\text { sociale et de la santé publique }\end{array}$ & sso & $\begin{array}{l}\text { Société suisse d'orthopédie } \\
\text { et de traumatologie }\end{array}$ \\
\hline & du Conseil des Etats & SSP & Société suisse de pédiatrie \\
\hline CSST & $\begin{array}{l}\text { Conseil suisse de la science } \\
\text { et de la technologie }\end{array}$ & SSPP & $\begin{array}{l}\text { Société suisse de psychiatrie } \\
\text { et psychothérapie }\end{array}$ \\
\hline СТM & $\begin{array}{l}\text { Commission des tarifs } \\
\text { médicaux }\end{array}$ & SSPTC & $\begin{array}{l}\text { Société suisse de pharmacologie } \\
\text { et toxicologie clinique }\end{array}$ \\
\hline FPPC & $\begin{array}{l}\text { Formation prégraduée, } \\
\text { postgraduée et continue }\end{array}$ & $\begin{array}{l}\text { SSRO } \\
\text { SVM }\end{array}$ & $\begin{array}{l}\text { Société suisse de radio-oncologie } \\
\text { Société vaudoise de médecine }\end{array}$ \\
\hline GTQ & Groupe de travail Qualité & VEDAG & Association des sociétés \\
\hline LAA & $\begin{array}{l}\text { Loi fédérale sur } \\
\text { l'assurance-accidents }\end{array}$ & & médicales de Suisse alémanique \\
\hline
\end{tabular}

